\title{
Graphene FET Sensors for Alzheimer's Disease Protein Biomarker Clusterin Detection
}

\author{
Theodore Bungon 1, Carrie Haslam ' ${ }^{1}$, Samar Damiati ${ }^{2,3}$, Benjamin O'Driscoll ${ }^{1}$, Toby Whitley ${ }^{1}$, \\ Paul Davey ${ }^{1}$, Giuliano Siligardi ${ }^{4}$, Jerome Charmet ${ }^{5}$ and Shakil A. Awan 1,* \\ 1 Wolfson Nanomaterials and Devices Laboratory, School of Engineering, Computing and Mathematics, \\ Faculty of Science and Engineering, University of Plymouth, Drake Circus, Plymouth, Devon, PL4 8AA, \\ UK; theodore.bungon@plymouth.ac.uk (T.B.); carrie.haslam@plymouth.ac.uk (C.H.); \\ benjamin.odriscoll@plymouth.ac.uk (B.O.); toby.whitley@plymouth.ac.uk (T.W.); \\ paul.davey@plymouth.ac.uk (P.D.) \\ 2 Department of Biochemistry, Faculty of Science, King Abdulaziz University (KAU), Jeddah, Saudi Arabia; \\ sdamiati@kau.edu.sa (S.D.) \\ 3 Division of Nanobiotechnology, Department of Protein Science, Science for Life Laboratory, School of \\ Engineering Sciences in Chemistry, Biotechnology and Health, KTH Royal institute of Technology, \\ Stockholm, Sweden \\ 4 Diamond Light Source, Rutherford Appleton Laboratory, Oxfordshire, OX11 0DE, UK; \\ siligardi.giuliano@diamond.ac.uk \\ 5 Institute of Digital Healthcare, WMG, University of Warwick, Coventry CV4 7AL, UK; \\ j.charmet@warwick.ac.uk \\ * Correspondence: shakil.awan@plymouth.ac.uk; Tel.: +44-(0)1752-586-325 \\ Published: 5 November
}

\begin{abstract}
We report on the fabrication and characterisation of Graphene field-effect transistor (GFET) Biosensors for detecting clusterin, a prominent protein biomarker of Alzheimer's disease (AD). There are approximately 54 million people currently living with dementia worldwide and this is expected to rise to 130 million by 2050. Although there are over 400 different types of dementia, $\mathrm{AD}$ is the most common type, affecting between $50-75 \%$ of those diagnosed with dementia. Diagnosis of AD can take up to 2 years currently using MRI, PET, CT scans and memory tests. There is, therefore, an urgent need to develop low-cost, accurate, non-invasive and point-ofcare $(\mathrm{PoC})$ sensors for early diagnosis of AD. The GFET sensors we are developing to address this challenge were fabricated on $\mathrm{Si} / \mathrm{SiO}_{2}$ substrate through processes of photolithographic patterning and metal lift-off techniques with evaporated chromium and sputtered gold contacts. Raman Spectroscopy was performed on the devices to determine the quality of the graphene. The GFETs were annealed to improve their performance before the channels were functionalized by immobilising the graphene surface with a linker molecule and anti-clusterin antibody. The detection was achieved through the binding reaction between the antibody and varying concentrations of clusterin antigen from $1 \mathrm{pg} / \mathrm{mL}$ to $1 \mathrm{ng} / \mathrm{mL}$. The GFETs were characterized using 4-probe direct current (DC) electrical measurements which demonstrated a limit of detection of the biosensors to be below $1 \mathrm{pg} / \mathrm{mL}$.
\end{abstract}

Keywords: Graphene; FET; biosensor; diagnosis; electrical detection; clusterin; Alzheimer's disease; dementia; protein biomarkers 